\title{
Mouse Free Cursor Control
}

\author{
Pooja C. Shindhe and Sangeetha Goud
}

\begin{abstract}
In this paper we present an idea for Human computer Interaction (HCI), where we have tried to control the cursor operation of the mouse using hand fingers (Red Green Blue). Hand gestures were collected using a camera based on color detection technique. This method mainly concerned on the use of a Web Camera to develop a virtual HCI device in a cost effective manner. The system that we aim to develop is interacting with humans i.e. it creates a human computer interface for Performing Mouse operations using $R G B$ plains. The geometrical operation and integral image processes are performed on the input. System will compare both coordinates and perform the action i.e. it will allow the user to control the mouse operations using webcam based on which color tape is used in front of the web camera.
\end{abstract}

Keywords--- Human Computer Interaction, RGB Plane.

\section{INTRODUCTION}

$\mathrm{H}$ UMAN beings usually depends on sense of vision. Vision makes human to understand the world surrounding them in proper manner. For our purpose, considering an image as a single picture which shows some information. It may be a picture of a person, or a thing. Pixels are tiny sections of color or tone that together form a photograph. Each pixel is made up of 3 values; red, green, and blue, or RGB. This RGB information is used to determine the color of each pixel. Vision-based tracking is very important problem in this field (HCI), since hand motions are potentially used to interact with computers in a natural ways. For example, video cameras and computer vision techniques may able to capture many details of human movement. The structure of the hand may be analyzed to manipulate an onscreen object in a way similar to the manipulation of paper on a desk. Such an approach may lead to a quick, and more natural style of interaction for certain tasks. Ubiquitous computing is devoted to changing the relationship between humans and the computers with which we interact, towards allowing computers to become invisible and recede into the periphery of people's lives. Here three colors are used to move the cursor and to click. In this study, finger tracking based a virtual mouse application has been designed and implemented using a regular webcam.

A click was implemented by defining a screen such that a click occurred when a user's hand passed over the region. Another method was developed where only the RGB objects

Pooja C. Shindhe, Department of Electronics and Communication, SDM College of Engineering and Technology, Karnataka, India. E-mail: poojashindhe90@gmail.com

Sangeetha Goud, Department of Electronics and Communication, SDM College of Engineering and Technology, Karnataka, India. E-mail: sangeethagoud@gmail.com

DOI:10.9756/BIJRCE.8210 were used to control the cursor operation. The clicking method was dependent on the color detected, and required the user to hold the colour finger on the desired spot for some period of time. Displacement of the red color finger or object while making a hand sign alters the mouse pointer. In this reference, a color pointer has been used for the object recognition and tracking. Left and the right click events of the mouse have been achieved by detecting the number of blue color objects on the image. In first case red color is used for mouse operation. In second case red plain is used to cursor pointing, one blue finger is used for right click, and two blue color finger for left click, and three blue color finger is used for double click, and green finger is used for scrolling.

\section{RELATED THEORY}

\section{A. Literature Survey}

In [1] intention was presenting the overview of HCI and Game Design in many aspects; the relationship between HCI and computer game, and the discussion of how HCI's processes and methodologies have been used in Game Design. In addition, the Thesis work followed the qualitative approach. Information was summarized and collected from a variety of published sources. The Thesis result concluded with there is a weak link between the HCI and the Game Design. For the reason, the idea of bringing HCI in the Game Design process is a new concept. Moreover, the Thesis also pointed out the important roles of the Heuristics in the Game Usability inspection. The intention paper [3] is to provide an overview on the subject of Human-Computer Interaction. The introduction includes the basic terminology and definitions, a survey of previous technologies and recent advances in this field, common architectures preferred in the design of HCI systems which includes multimodal and uni modal configurations, and finally the HCI applications. This paper also offers a comprehensive number of references for each concept, method, and application in the HCI. In [4] an efficient method is presented involving gesture and voice based interactive experiments to simulate mouse functions. There are many other interactive HCI systems become very popular in recent era. The approaches involved in the present method comparing the earlier one used for mouse simulation are hardware system. This approach tries to eliminate the use of traditional way of controlling mouse. In the approached method a web cam for the acquiring images and to analyze various gestures recognition techniques are used for mouse operations. This system has many applications in entertainment, commercials fields. Another paper [5] presents a new method for handling mouse operation using a real-time camera. Earlier methods involve replacing the mouse parts. Instead of changing the parts a new method is intrudes to change the hardware design. This method consider a camera and computer vision technology, such as gesture recognition 
and image segmentation, to handle tasks (clicking, doubleclicking, and scrolling) and show how it can perform everything a new mouse devices can. This paper describes how to build present cursor control system. In [6] paper, novel approach for Human Computer Interaction (HCI) is proposed where, cursor operations using a real-time camera is carried. Current method replaced with a new method to use a camera and computer vision technology, for controlling mouse tasks (left and right clicking, double-clicking, and scrolling) and also show how it can perform everything as current mouse devices can. The software is developed in JAVA language. Pose estimation and Recognition in this approach are free from user and coloured fingers are used to perform actions. The software also preferred as an intuitive input interface to the system that needs multi-dimensional control e.g. computer games etc.

\section{B. Block Diagram}

Figure 1 shows the block diagram of the proposed work. The first step of our system is to differentiate the potential pixels from the non-potential pixels. This can be done by subtraction scheme of image background which segments any potential foreground hand information from the background scene. At the start-up, a couple of background video is captured to shows the static workspace from camera view. Then a subsequent frame uses the appropriate background of a image to segment out moving foreground data. In the object tracking method one of the major objectives is object identification. A color pointer has been used to make the object detection quick and ease. A red stickered finger is used as a pointer in this approach. To perform the click events of the mouse, finger with blue color pointers has been used. The algorithm of the system is as follows:

1. Placing the pointer.

2. Indentify the pointer using defined color information

3. Marking the region and defining the centre of the pointer by drawing a bounding box.

4. Tracking the moment of the pointer.

5. Placing the cursor according to the centre of the pointer.

6. Execute the single and double click and the right and left click of the mouse.

Acquiring the real time vido from web cam

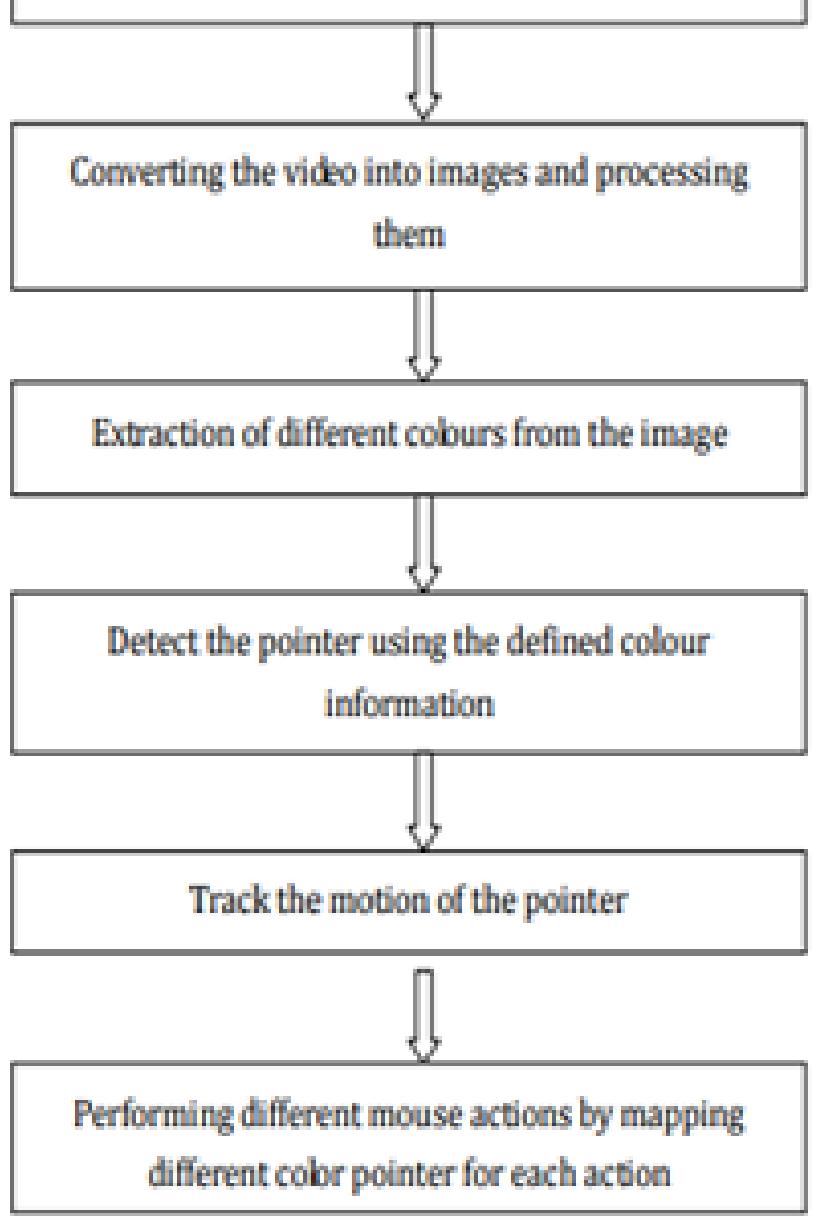

Figure 1: Block Diagram of Proposed Work 


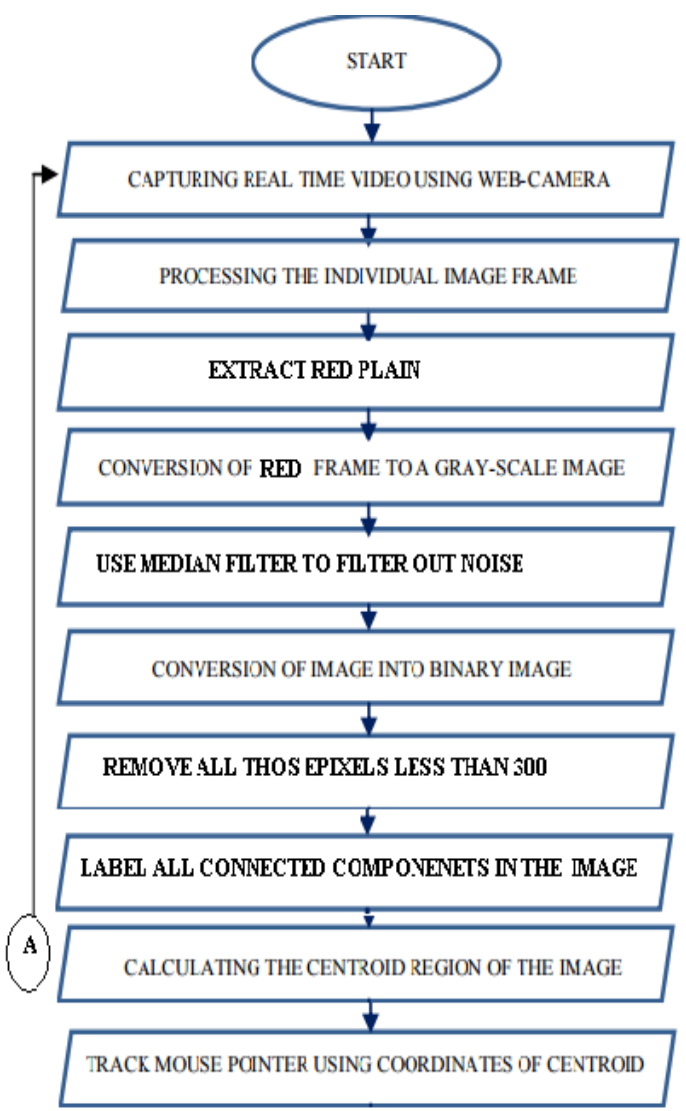

Figure 2.a: Detection of Red Component

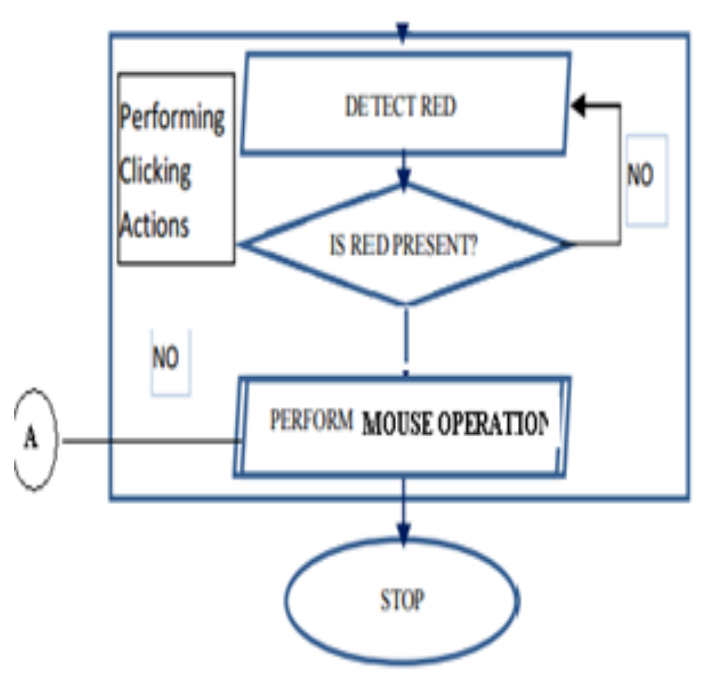

Figure 2.b: Detection of Red Component

Figure 5.2 shows the block diagram used to detect the red color which is used in the proposed work to move the cursor. The cursor can be controlled by using the various colors .In this project red color is used. R plane, initially video capturing is done. here the real time video is captured by using the built in web cam and then from the real time video ,individual image frames are obtained using the instruction "getsnapshot". Then we need to obtain the red plane from the images using the instruction "imsubtract". To obtain the red plane for the image the threshold is applied.
To make the binary scale image from a gray scale image a MATLAB's built in function "im2bw" is used. Function can be used as; BW = im2bw (I, level) it helps to convert the gray scale image in to a binary image. The output of the operation sets all pixels in the input image which are greater than luminance level with the value 1 (white) and sets all other pixels with the value 0 (black). That specify levels in the range $[0,1]$. This range is similer to the signal levels possible for the image's class. Therefore, a level value of 0.5 is midway between black and white, regardless of class. In our study, the threshold 0.15 gave the best result for the large range of illumination change as shown in figure 3 . Then obtained image is given to median filter to eliminate salt and pepper noise using instruction "medfilt2". (a)

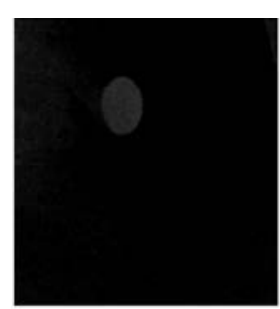

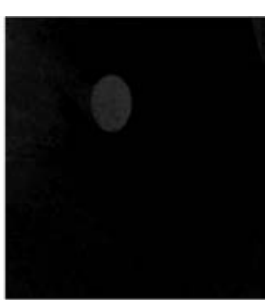

(b)
Figure 3 (a): Before Median Filtering (b) After Median Filtering

To detect color from the image, MATLAB's built in "imsubtract" function is used. Imsubtract function can be used as; $\mathrm{Z}=\operatorname{imsubtract}(\mathrm{X}, \mathrm{Y})$ it subtracts each $\mathrm{Y}$ array element from the corresponding $X$ array element and gives the difference in the corresponding $\mathrm{Z}$ array element. It uses the $\mathrm{Z}$, $\mathrm{X}$ and $\mathrm{Y}$ as real and non-sparse numeric arrays of the same class and size. $\mathrm{Y}$ is a double scalar. Later the image is converted to binary form so that processing speed increases and the computation becomes easy as shown in figure 5.6 using instruction "im2bw". Then the centroid calculation is carried out so that we get $\mathrm{X}$ and $\mathrm{Y}$ co-ordinates, so that whenever pointer moves we get the actual co-ordinates at that position. Now we need to interface the virtual mouse to carry out the operations of the actual mouse to the computer ,this is done by importing java package, since matlab doesn't have built in function to interface and then the operation is completed.

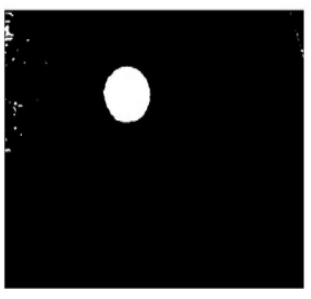

(a)

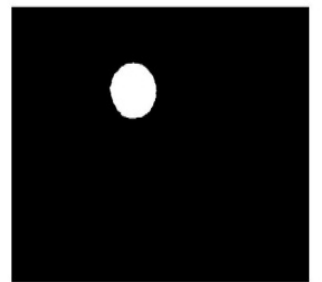

(b)
Figure 4: (a) Small areas Before the Removal (b) Small Areas After the Removal

Subtracting all the Small Areas to get the actual accurate number of object indentified in the image, all the other areas excluding the pointer need to be removed. To perform these, use MATLAB's "bwarea open” function. It is shown in figure 5.6. 
Function can be used as" BW2 = bwarea open (BW, P)" it removes a components from binary image that all having fewer than P pixels, produces another binary image, BW2. A 300 pixels threshold is set for the approach. Figure 5 shows the sequence of operations that are carried out in order to perform all the mouse events viz. click and scroll.

Initially the real time video is captured using web camera, after that the individual frames out of the captured video will be processed then flipping of the image will be done. Flipping is done to remove the red region which has arrived out of the bounding region. Immediately after this the color detection and extraction of different colors is done and converted to gray scale image after that we convert it to binary image so that computation becomes easy and processing becomes faster. Once the image is converted to binary we calculate the centroid of the image so that we can locate the pointer. Accordingly as we move the red plane we can track the pointer using co-ordinates of the centroid. Further it will detect if any red object is present, if present it will perform the tracking of pointer as we move the red object accordingly there will be movement of the pointer, otherwise it will be scanning for red colour objects. Further it checks for blue objects, if blue object is present it will search for number of blue objects. If one blue object is present it will perform left click, if two blue objects are present it will perform right click and if three blue objects are present it will perform the double click operation. Finally it will check for green colour objects, if present it will perform up and down scrolling based on the movement of the green object.

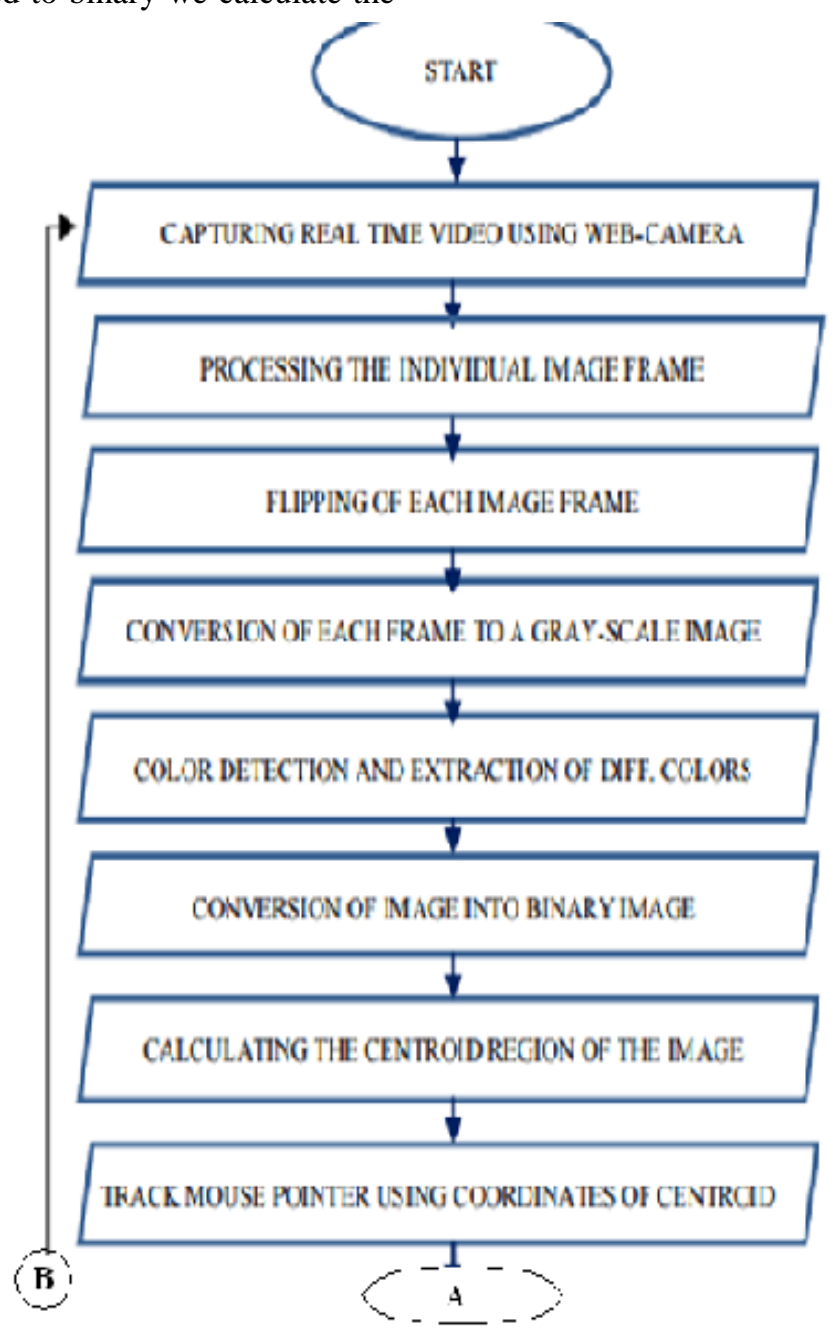

Figure 5.a: Sequence of Mouse Operations

The red color taped finger used as a cursor controller to control cursor movement. Two types of methods for moving the cursor can be used. The first one is position mapping the finger on a screen of the camera, to a position on the desktop screen. But this method faces a problem. That is if the camera resolution is lesser than the resolution of the desktop window, then because of losing the intermediate values the cursor position cannot be accurate while converting camera to the desktop window resolution. The expected ratio of jumping pixel is up to 4 pixels. The second one is weighted speed cursor control. Here the differences between the two images are considered and the distance between the two is compared. Next, the mouse cursor is moves fast if the distance between the images (current and previous frame) is far or, if the distance is close then the cursor moves slow. This algorithm is also faces a problem. Some hardware which cannot achieve image processing more than 15 fps do not work smoothly because computing the image centre and the hand shape takes time. 
Finding the Centre of the Object and putting the Bounding Box After cancelling all the connected components (objects) excluding the pointer, to label the pointer MATLAB's "bwlabel" function is used. To get the properties of the region such as centre point or bounding box etc., MATLAB's built in "regionprops" function can be used as; STATS =
regionprops(BW, properties) It measures a set of properties for each connected component (object) in the binary image, $\mathrm{BW}$. The image BW is a logical array; it can have any dimension. In our application since we need to get the bounding box and the centre of the object, we need both properties.

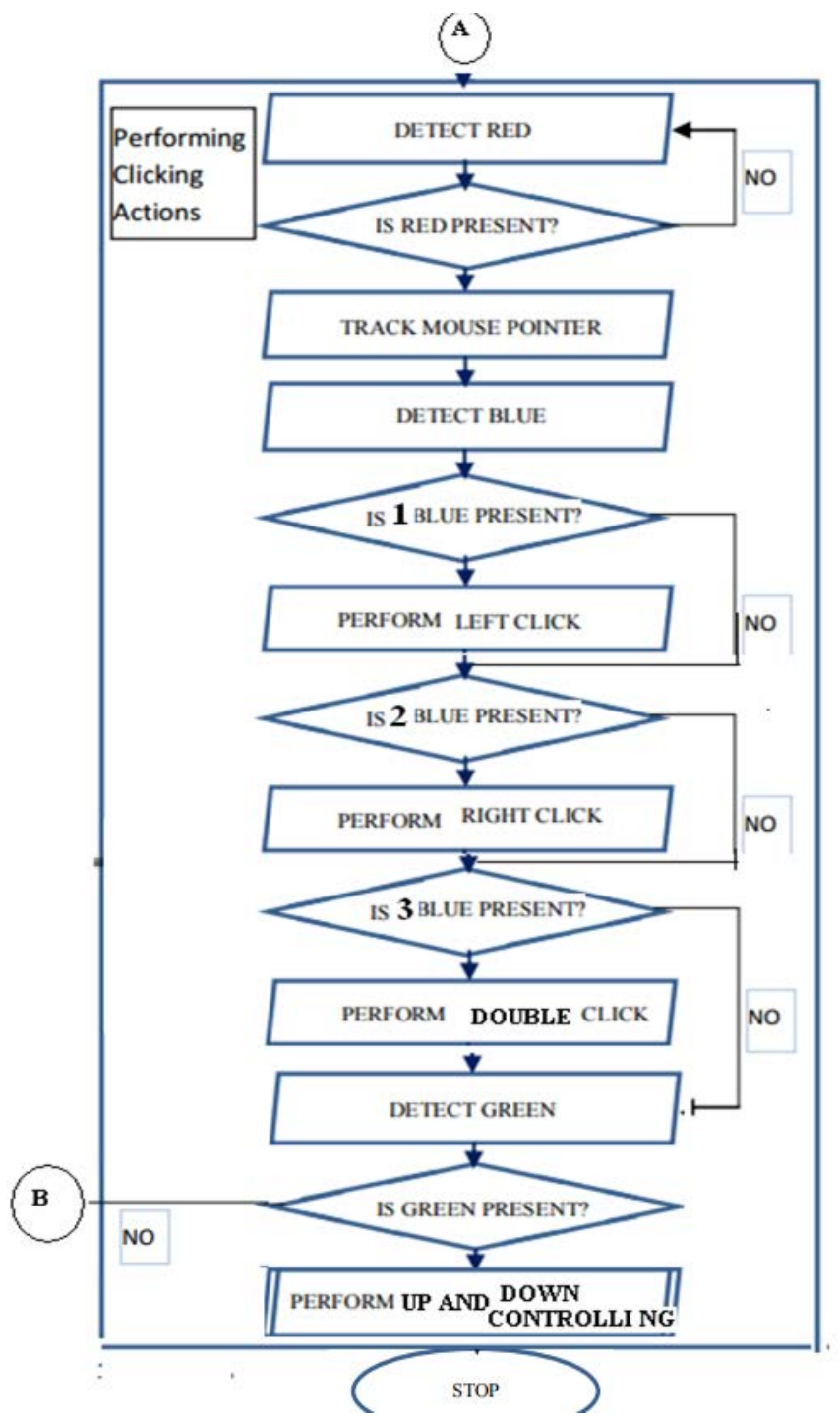

Figure 5.b: Sequence of Mouse Operations

\section{MOUSE OPERATION}

\section{A. Tracking the Mouse Pointer}

Once the coordinates has been identified, the driver of the mouse is accessed and the coordinates are dispatched to the cursor. Using these coordinates, the cursor places itself in the required position. It is assumed that the object moves continuously, to create an affective tracking every time a new centroid is determined and for every frame the cursor obtains a new position. So as the user moves his hands across the camera field, the mouse moves proportionally across the screen. . A java object is created and it is linked with the mouse driver. Because there is no inbuilt function in MATLAB to directly access the drivers of the computer. MATLAB supports integration with other languages like C,
$\mathrm{C}++$, and JAVA. Since java is a machine independent language so it is preferred over the others. Based on the detection of other colors along with red the system performs the clicking events of the mouse. These color codes can be customized based on the requirements.

\section{B. Performing Clicking Actions}

The flags associated with the mouse buttons are used to control actions of the mouse. To access these flags JAVA is used. The user needs to perform some of gestures in order to create the control actions. Due to the use of color pointers, the computation time required is reduced. Furthermore the system becomes resistant to background noise and low illumination conditions. The identification of the colors follows the same procedure. Clicking action uses the simultaneous detection of both red and blue colors. 


\section{Color Fingers to Use.}

To execute the cursor events the following colors are used in the proposed work.

1. To control the POINTER POSITION move the RED finger everywhere.

2. LEFT CLICK : Show ONE BLUE finger

3. RIGHT CLICK : Show TWO BLUE finger

4. DOUBLE CLICK: Show THREE BLUE finger

5. MOUSE SCROLL: MOVE the GREEN finger up and down.

Mouse click is one of the most important parts for the virtual mouse. The left button of the mouse has a different task for different clicks. When the second pointer is identified in the image, left mouse click event performed. When the second pointer is being indentified both clicks have been executed.

\section{Red Object Detection}

In the RGB plane Red color object is used to control the pointer position. Here the red color tapes are used as shown in figure 6 .

\section{a) Green Plane Detection}

In the RGB plane green color object is used to control the mouse scroll .Here the green color tapes are used as shown in figure 7 .

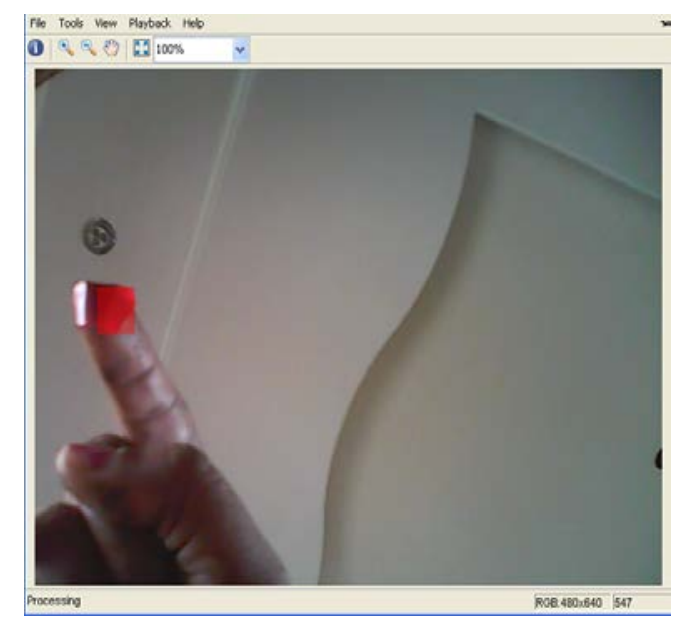

Figure 6: The Red Tapes used During the Control of Pointer

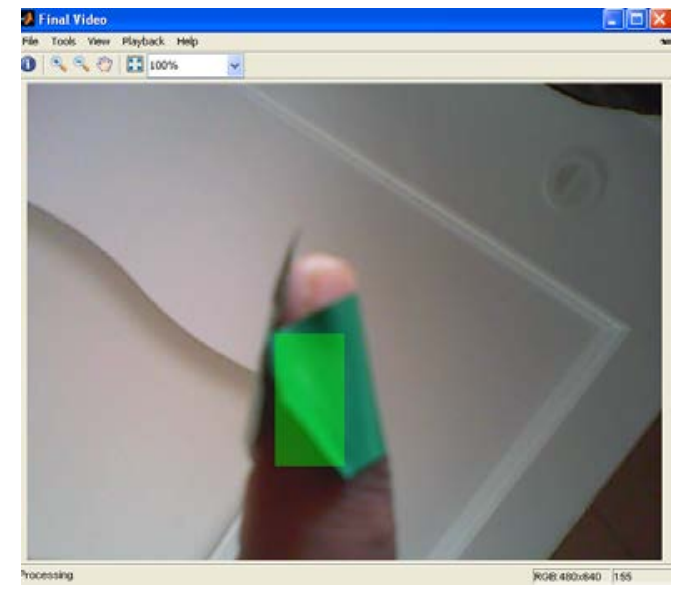

Figure 7: Indicate the Red Tapes used During the Scrolling of Pointer

\section{b) Blue Plane Detection}

In the RGB plane blue color object is used to perform the clicking operation .Here the blue color tapes are used as shown in figure 8.

\section{c) RGB Plane Together Used during the Operation}

These images i.e. figure 9 indicate that all the three colors are stick to three different fingers, so that the operation becomes more flexible.

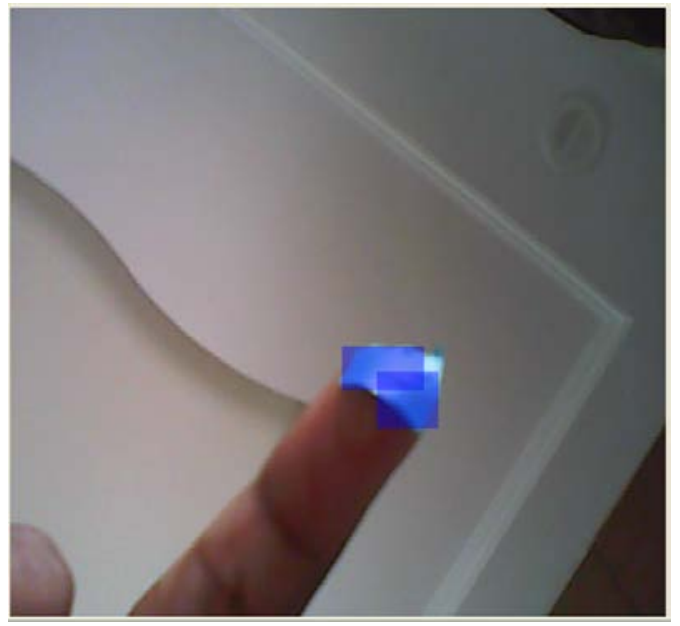

Figure 8: The Blue Tapes used During the Clicking Operation

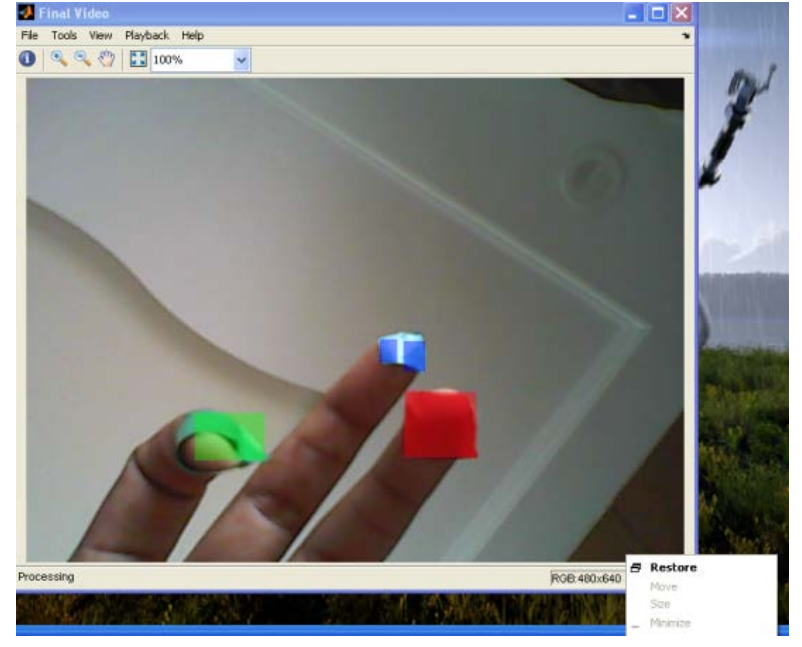

Figure 9: All the Three Color Stick to Same Hand

\section{CONCLUSION AND FUTURE WORK}

In this project, a virtual mouse application which is basically objects tracking is developed and implemented using a web camera. The system has been executed in MATLAB software using Image Processing Toolbox. A blue color sticker is used as an object to make the detection easy and fast. Both object identification and moment tracking worked well. Using the pointer moving the cursor and the simulating the mouse click events also worked well. However, system has some disadvantages such as; being invariant to illumination up to some scale, and movement of the cursor is very sensitive to motion. Because of this reason, to control the cursor, pointer cannot be used on the air efficiently. 


\section{ACKNOWLEDGEMENT}

I thank Prof. S.V. Viraktamath, PG Co-ordinator, Department of Electronics and Communication Engineering for his timely suggestions and support. He has been especially enthusiastic in giving his opinions and critical views.

\section{REFERENCES}

[1] H. Nguyen, "Human Computer Interaction in Game Design”, 2012.

[2] J.R. Parker, "Algorithms for Image Processing and Computer Vision", Wiley Publishing ,Second Edition, 2011.

[3] F. Karray, M. Alemzadeh, J.A. Saleh and M.N. Arab, "Human-computer interaction: Overview on state of the art”, Vol.1, No.1, 2008.

[4] S. Pratik, "Gesture based interactive system”, IJIRS, Vol. 2, No.3, 2013.

[5] H. Park, "A Method for Controlling the Mouse Movement using a Real Time Camera”, Brown University, Providence, RI, USA, Department of computer science, 2008.

[6] N. Kamran, "Mouse Simulation Using Two Colored Tapes", International Journal of Information Sciences and Techniques (IJIST) Vol.2, No.2, 2012.

[7] R.C. Gonzalez and E. Richard, "Woods, digital image processing”, ed: Prentice Hall Press, 2002.

[8] J. Accot and S. Zhai, "Beyond Fitts' law: models for trajectory-based HCI tasks", Proceedings of the ACM SIGCHI Conference on Human factors in computing systems, Pp.295-302, 1997.

[9] H. Park, "A Method for Controlling the Mouse Movement using a Real Time Camera”, Brown University, Providence, RI, USA, Department of computer science, 2008.

[10] Q. Li, U. Niaz and B. Merialdo, "An improved algorithm on Viola-Jones object detector", 10th International Workshop on Content-Based Multimedia Indexing (CBMI), Pp. 1-6, 2012.

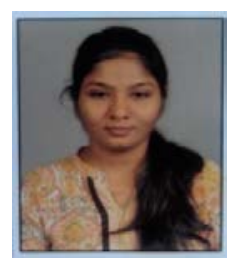

Pooja C. Shindhe received the BE degree from the Department of Electronics and Communication Engineering, VDRIT, Haliayal, in 2015. She is currently doing Masters degree in the Department of digital electronics, SDMCET Dharwad Karnataka, India. She presented paper on the topic of "Review on 5 G Network" in national conference held in Belgaum in 2015.

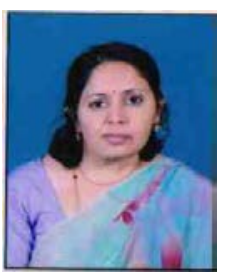

Sangeetha Goud (Dharwad). Received the MBA and M.Tech degree from MISIT. Working as professor in SDMCET Dharwad. 